\title{
Flares Detected on Some Late Giants: Are They Real?
}

\author{
R. Konstantinova-Antova ${ }^{1}$, M.M. Ivanov ${ }^{2}$, A.P. Antov ${ }^{1}$ \\ 1 Institute of Astronomy, Bulgarian Academy of Sciences, 72 Tsarigradsko shose, BG- \\ 1784 Sofia, Bulgaria \\ 2 Department of Astronomy, Sofia University, 5 James Bourchier Av., BG-1126 Sofia, \\ Bulgaria
}

\section{Introduction}

Recently, some apparently single red giants with evidence of chromospheric activity were reported. In this work we observed those single late giants with reported flares, referenced by Pettersen (1989) and Schaefer (1989), as part of a program for investigation of the chromospheric activity in $\mathrm{G}$ and $\mathrm{K}$ giants.

According to Simon \& Drake (1989), active single $G$ and $K$ giants may be first crossing stars that have just entered the phase of rapid spin-up as their convection zones advance upon their more rapidly rotation radiative cores. These stars are situated on the base of the red giant branch on the HR diagram. As a result they may possess dynamo driven activity at this stage of evolution.

\section{Observations and Results}

The aim of our research is to investigate suspected chromospherically active late giants and especially those with reported flares, using some typical indicators, accessible with our equipment, i.e. $\mathrm{Ca} I \mathrm{II}+\mathrm{H}, \mathrm{H} \alpha$ and flares (Ulmschneider 1979).

For this purpose, spectroscopy and photometry were carried out for $\nu$ Oph (G9 III), $\tau$ CrB (K0 III) and V654 Her (K2 III). The $2 \mathrm{~m}$ telescope at the National Astronomical Observatory at Rozhen, equipped with a Coude spectrograph and CCD detector was used along with two $60 \mathrm{~cm}$ telescopes equipped with singlechannel photon-counting photometers and a $U$-filter. The integration time was $1 \mathrm{sec}$.

High-resolution spectra were obtained for each star on more than one night with $\mathrm{S} / \mathrm{N}>100$ at $\mathrm{H} \alpha$ and $\mathrm{S} / \mathrm{N}>50$ at $\mathrm{Ca}$ II $\mathrm{K}+\mathrm{H}$. No evidence of chromospheric activity, such as emission cores in $\mathrm{Ca}$ II $\mathrm{K}+\mathrm{H}$ were observed (Fig. 1). $\mathrm{H} \alpha$ was in absorption and seems not to be filled-in (Fig. 2). $\mathrm{H} \alpha$ spectra of OP And, an apparently single, chromospherically active $\mathrm{K} 1$ giant, were obtained with the same equipment. Its $\mathrm{H} \alpha$ absorption is partially filled-in and, according to Strassmeier et al. (1990), it also exhibits strong Ca II $\mathrm{K}+\mathrm{H}$ emission. 
V654 Her is too faint for us to obtain a spectrum in Ca II $\mathrm{K}+\mathrm{H}$, so we used those presented in Tsvetkov \& Pettersen (1985). Their low-dispersion spectrum shows no emission cores.

$17^{\mathrm{h}} 52^{\mathrm{m}} 34^{\mathrm{s}}$ total monitoring time was obtained for $\mathrm{V} 654$ Her and $13^{\mathrm{h}} 53^{\mathrm{m}} 44^{\mathrm{g}}$ for $\tau \mathrm{CrB}$. No flares were detected. $\nu$ Oph was too bright to be observed with our photometric equipment.
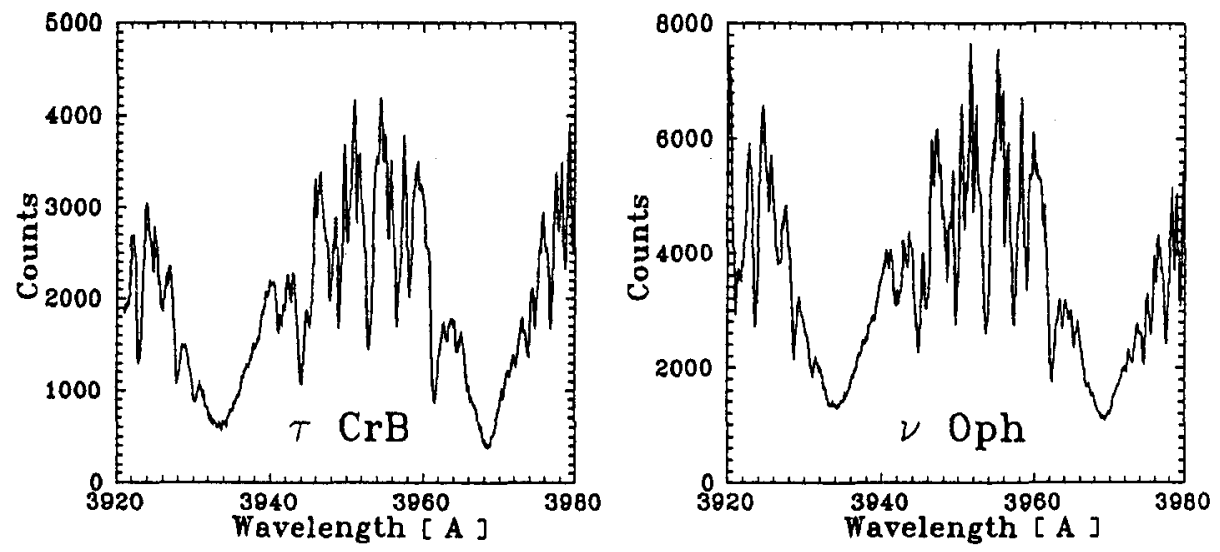

Fig. 1. Spectra of $\nu \mathrm{Oph}$ and $\tau \mathrm{CrB}$ in the region of $\mathrm{CaII} \mathrm{K}+\mathrm{H}$.

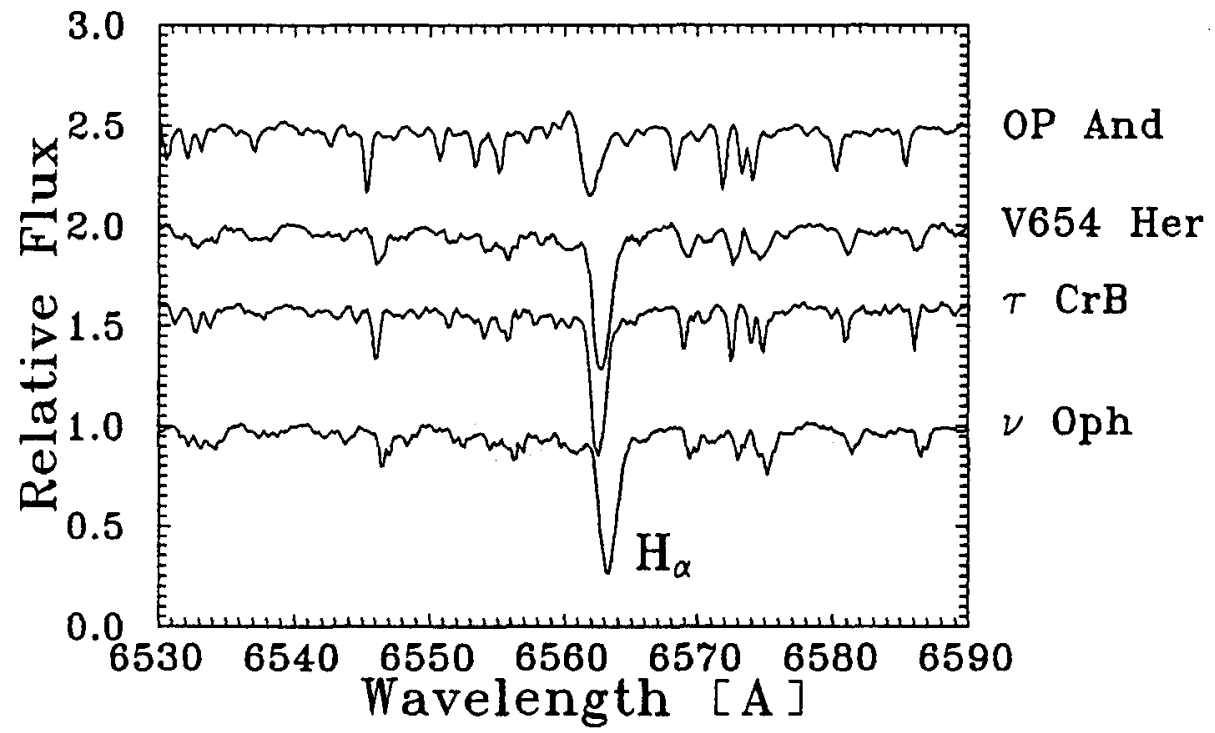

Fig. 2. $\mathrm{H} \alpha$ spectra of the stars investigated. OP And is also presented for comparison. 


\section{Discussion}

Our work revealed a lack of typical chromospheric activity indicators in all three stars. In Fig. 3 we show a HR diagram derived using the evolutionary tracks of Schaller et al. (1992) for solar abundance and $M_{\text {bol }}$ and $T_{\text {eff }}$ from Allen (1973).

$M_{V}$ and $B-V$ for $\nu \mathrm{Oph}$ and $\tau \mathrm{CrB}$ were taken from Wilson (1976). The $M_{V}$ of $\tau$ CrB differs significantly from other $\mathrm{K} 0$ giants and so we took its $M_{\mathrm{V}}$, obtained from DDO photometry by Brown et al. (1989). For V654 Her we used $B-V$ value obtained by us (a mean value from 8 nights of observation at the Belogradchik Observatory) and $M_{V}$ was derived using standard models from Allen (1973). The stars are situated high above the base of the giant branch and thus are inconsistent with Simon \& Drake's (1989) hypothesis.

These stars seem to be normal giants and cannot be considered as chromospherically active. The nature of the events reported in Pettersen and Schaefer and presented in Table 1 remains a puzzle. We point out that the so-called flares were detected only once, and no other have been observed since.

If we analyze the reported flares we come to the following conclusions:

1. The event, detected on $\tau \mathrm{CrB}$ visually, may have been due to a satellite glint, meteor or other sky effect.

2. The same might be possible in a lesser degree for the flare on $\nu \mathrm{Oph}$.

3. The nature of the so-called flare, detected photometrically on V654 Her, is unclear. We consider the star as chromospherically inactive. But we don't know artificial causes which might simulate a flare. Since the event seems not to be due to chromospheric activity, its nature remains unknown.

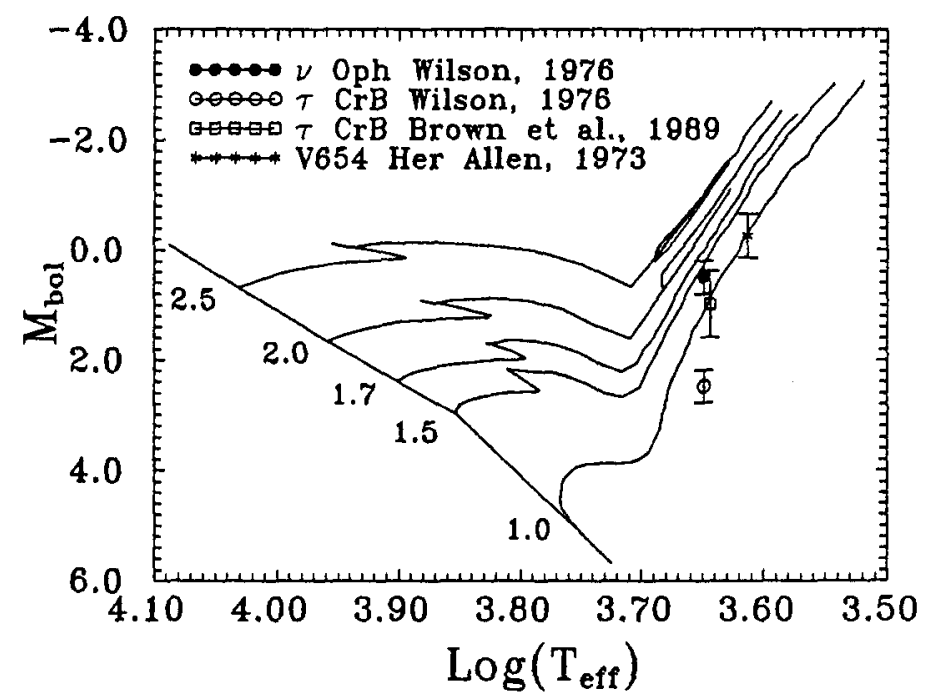

Fig. 3. Positions of the investigated stars in the HR diagram. 
Table 1. The table is taken from Schaefer (1989), where: $\Delta m$ is the amplitude of the event; $t$ is the minimum $e$-folding rise or fall time; $D$ is the total event duration; $N$ is the number of independent measurements which confirm the existence of an anomaly; and $q$ is the angular resolution of the detector.

\begin{tabular}{ccccccccc}
\hline Star & Sp. Type & $m_{V}$ & $\Delta m$ & $t(\mathrm{sec})$ & $D(\mathrm{sec})$ & $q(")$ & Detector \\
\hline$\nu$ Oph & G9 III & 3.3 & 7 & 10 & $>80$ & 10 & 300 & visual \\
$\tau$ CrB & K0 III & 4.7 & 1.7 & $<0.25$ & 0.5 & 1 & 300 & visual \\
V654 Her & K2 III & 10.0 & 0.15 & 140 & 300 & 50 & 10 & photometer \\
\hline
\end{tabular}

Acknowledgements: The authors are thankful to Drs. N. Tomov and R. Zamanov for their help in obtaining the spectroscopic material. R.K.-A. is grateful to the German Science Foundation for financial support to attend the Colloquium. She is also thankful to Dr. R. Pallavicini for useful discussions. This work is partially supported by the Bulgarian National Science Foundation under contract F-347/1993.

\section{References}

Allen C.W., 1973, Astrophysical Quantities, Athlone Press, London, p. 289ff.

Brown J.A., Sneden Ch., Lambert D.L., Dutchover Jr. E.., 1989, ApJS 71, 293

Pettersen B.R., 1989, Solar Phys. 121, 299

Schaefer B.E., 1989, ApJ 337, 927

Schaller G., Schaerer D., Meynert G., Maeder A., 1992, A\&A 96, 269

Simon T., Drake S.A., 1989, ApJ 346, 303

Strassmeier K.G., Fekel F.C., Bopp B.W., Dempsey R.C., Henry G.W., 1990, ApJS 72,191

Tsvetkov M.K., Pettersen B.R., 1985, A\&A 150, 160

Ulmschneider P., 1979, SSR 24, 71

Wilson O.C., 1976, ApJ 205, 823

P.B. Byrne: The closest analogy to active giants are the RS CVn stars. There, optical flares are rare. So, is $14-18$ hours monitoring sufficient to rule out optical flares?

R. Konstantinova-Antova: Of course, more observations are required, because the giants are more luminous than the dwarf flare stars. Also, we don't know whether the dynamo mechanism, suspected to operate on them is with a comparable efficiency as on the active dwarfs. But for the three giants I reported, there are no other evidences for chromospheric activity than the fact that they exhibited (in two of the cases under suspect) such flashes. In the light of our investigations (including spectral ones) I cannot say that they are due to chromospheric activity. 